\title{
Escape to Nasopharynx During Swallowing
}

National Cancer Institute

\section{Source}

National Cancer Institute. Escape to Nasopharynx During Swallowing. NCI Thesaurus.

Code C127228.

A finding of escape to nasopharynx during swallowing. 